\title{
DMT - an integrated disaster management tool
}

\author{
M. Angermann, M. Khider, M. Frassl \& M. Lichtenstern \\ German Aerospace Center (DLR), Germany
}

\begin{abstract}
This paper describes a system aimed at realizing an efficient flow of information to and from all involved parties in disaster response missions. The system is called Disaster Management Tool (DMT). It is comprised of hardware and software and tightly integrates satellite based rapid mapping, communication and navigation. We describe the background, motivation and objectives that are guiding its development as well as details on the system design and implementation. The system has been through a number of field tests. In particular we give experimental insights obtained from participating in the Assessment Courses (AMC) on Cyprus in 2008 and 2009. The DMT is a work in progress and we give an outlook on the future foreseen developments.

Keywords: disaster management, location based services (LBS), rapid mapping, humanitarian relief, situation assessment, information management, mapcase, NASA World Wind.
\end{abstract}

\section{Introduction}

\subsection{Background}

Time and information are the scarcest resources when disaster strikes. The effectiveness of the response depends on timely and reliable information. It is often difficult to determine what kind of support is needed and can be sent, since in most sudden onset natural disasters the overall situation is complex. Information on the location of the event, damage, affected population and the transport infrastructure still available are critical to decide on the extent, composition and deployment of international disaster response resources. Consequentially, satellite based rapid mapping, communications and navigation are becoming increasingly important as 
tools that provide valuable information for planning and operational support of humanitarian relief efforts during crisis situations.

Recent work has indicated the potential of rapid mapping and satellite based communication services as useful tools. This is reflected in initiatives like the International Charter 'Space and Major Disasters' or RESPOND (http://www.respondint.org/respondlive), an alliance of European and International organisations working to improve access to maps, satellite imagery and geographic information for the humanitarian relief community. Communication between relief forces in the field is essential for coordination as is dissemination of aggregated situation information into the field.

\subsection{Motivation and objectives}

Today, most relief organizations employ satellite communication networks, for voice and data communications between on-site and central headquarters. Today, receivers for GPS are widespread and provide personal navigation in an often unfamiliar environment. Today, earth observation satellites provide overhead imagery, mostly used in headquarters for strategic decisions. Today, the potential of combining the individual systems to an integrated infrastructure is only utilized to a minimal extent.

However, if done well, this integration can provide huge benefits by realizing an efficient flow of information to and from all involved parties in a mission and - most importantly -freeing personal in the field from performing too many secondary technical tasks involving too many isolated technical systems and software applications, thereby letting them concentrate on the primary aspects of their mission.

Our work addresses the existing lack of an integrated and rapidly deployable system in order to support the efforts of relief missions in disaster management. We have been working on such rapidly deployable systems within recent and ongoing projects. In this paper we are presenting the latest result of our work, the "Disaster Management Tool - DMT".

The DMT has been partially developed in the framework of an integrated project co-funded by the European Commission called LIMES (Land and Sea Monitoring for Environment and Security). Our work has been influenced by the following objectives set forth in the project:

- Advance the coordination and data sharing of crisis relevant information within the European and international context to minimize time losses between data acquisition and information extraction

- Development and integration of dedicated telecommunication tools in crisis management for robust bi-directional exchange of information between headquarters and field teams, including transfer of vector/raster information for situation assessment as well as robust communications and information exchange between teams in the field

While these objectives have set the initial scope of our work, our methodology is shaped by a quality criterion that is simple to formulate but demanding to 
achieve: A system that is fully accepted by the disaster management community for its usability and usefulness.

The litmus test for fulfilling this criterion would be the integration of the DMT into the standard equipment of relief teams, such as sent out on urgent response actions under the European Community Mechanism for Civil Protection or UNDAC (United Nations Disaster Assessment and Coordination) teams sent by UN OCHA (United Nations Office for the Coordination of Humanitarian Affairs) for similar purposes.

In this paper, background information about the DMT, its purpose and functionality, development methodology and requirements, system design and implementation aspects (software and hardware) are explained. Additionally, details on the communications and navigation tools, the rapid mapping concept and the different hardware flavours are illustrated. Field experiences and user feedback from our field tests of the DMT in the Assessment Mission Course (AMC) in Cyprus in 2008 and 2009 are presented. Finally, conclusions and an outlook of the future work towards extending the system's capabilities will be given.

\section{Methodology}

Since our quality criterion is user acceptance, users have been closely involved, starting from the initial concept and are consulted continuously for feedback. Since a DMT is targeted at being used in an extremely complex environment, we have found that it is not suitable to design a system in a traditional "requirements, design, implementation, test (against requirements)" approach. This is mainly caused by the fact that most relevant users have not yet experienced the functionality as to be provided by the DMT and have difficulties in explicitly formulating their requirements.

As a consequence of this, we started with the analysis of previous relief missions and rescue operations [1, 2, 3] and had a look on already existing proposals [4]. Based on our initial insights obtained by this analysis we led interviews with experienced disaster management experts to infer an initial list of important requirements that likely have to be met by our system, in order to become useful. As an example of striving to meet such requirements, the system is designed for "zero-configuration" upon setting up when arriving at an incident. We then participated as observers in a number of exercises and missions, "looking over the shoulder" of the experts, which a) gave us a lot of new ideas and b) taught us that many of our initial ideas would not be practical under field conditions.

Based on these initial "barehanded" field experiences we started to design and implement a prototype, capable of providing initial, not yet fully integrated services, such as satellite communications, displaying of maps, or georeferenced photography. With this prototype we were able to study the use of its features under field conditions, rapidly adapt and study again and again. We found that this method leads to satisfied (test) users, since they experience that their input is valuable and actually affecting the system and assures that development effort is spent on desired functionality and usability. Since these initial days we have 
kept this approach of small step improvements and direct incorporation of user feedback and ideas.

\section{Requirements}

The disaster relief organizations have numerous geographic information requirements depending on which sectors they are involved. Nevertheless, several common needs prevail: All organizations require the geographic information to be accurate, objective, timely, and in a suitable, standardized format. There is a need for maps as well as for quantified information like the extent of a flood, the remaining resources or the location and condition of damaged infrastructures.

Communication is particularly essential in case of humanitarian relief and often an issue due to the lack of proper communication lines, which may be destroyed. Consequently, each relief organization has its own telecommunication setup. This can range from standard broadband internet, telephone or fax to more basic VHF (Very High Frequency Radio) coverage and more costly satellite communication. But a reliable system that combines several communication technologies is still missing. Such a system would help relief organizations to communicate independently from a specific infrastructure that might be destroyed.

The requirements can be grouped in two parts: Field communication and information management and Localization and context determination.

\subsection{Field communication and information management}

Field communication and information management is considered vital for the operations. Required capabilities and constraints depend on the deployed unit and the actual mission. There are for example significant differences between longterm projects and short term emergency response missions. Due to the difficulties associated with mobilizing personal in the disaster area, no communication experts might be available to configure or operate communication components in the field. This results in a strong requirement on self-configuration of all communication components. Previous communication and information management solutions have been offered and tested by users such as the German Federal Agency for Technical Relief (THW). These tests have shown that communication and information management systems should be able to operate autonomously, without critically relying on a central infrastructure. Negative examples are web-based clients that require data transfer via satellite to a central server for every user action, thus resulting in intolerable delays and poor availability. Electronic devices should be robust against shocks from transportation and rough handling but are typically operated in sheltered place such as a tent or building. Therefore, no extreme precautions against water (heavy rain) or dust have to be integrated. 


\subsection{Localization and context determination}

Current operational procedures foresee the daily filing of travel plans for all vehicle based activities. However, these filings include only planned routes and activities and do not capture the actual state which may divert significantly from the plan. Furthermore, the traditional method depends on the discipline of all personal to file travel plans. Precise and automatic location reporting in combination with suitable map-based dynamic displaying of persons' positions and eventually their other context, such as current work load, stress, vital parameters, fatigue, capabilities or perceived threats could help to organize the operations and improve safety and security.

Automatic geo-referencing of important infrastructures or on-site findings, such as INSARAG (International Search and Rescue Advisory Group) markings that capture information relevant for search and rescue operations after earthquakes (e.g. a building's structural condition or information on missing or rescued inhabitants) is considered to be potentially very helpful for assigning resources. However, it is important to maintain "backward compatibility" with traditional means, in this case spraying the markings on a wall using ordinary spray cans.

\section{System design}

The purpose of the system is to efficiently combine communication, navigation, information management and earth observation data to achieve a minimal configuration - maximal usability tool and valuable support for relief workers.

\subsection{The mapcase concept}

Our basic system component are the so called mapcases. A mapcase is a robust plastic box including several pieces of hardware, assembled to work hand in hand with our software, the DMT. The mapcase allows mobile field workers and control center officers to easily submit and access information about their areas of interest. Doing so, it allows a fast and comprehensive exchange of situation awareness. Examples of distributed information are maps, points of interest (POIs), assessment reports, geospatial information like team positions, information about specific areas or geo-tagged images.

Additionally, the aim of the mapcase is to simplify the configuration and employment of communication hardware and sensor equipment, assisted by the software running on the system. The user can concentrate on his original tasks and does not have to waste time caring about technical issues.

We developed three types of mapcases for different use cases: The Stationary Advanded Mapcase (SAM), the Mobile Mapcase (MoM) and the Personal Assistant Mapcase (PAM, which is a wearable unit). They differ mainly in their size and the amount of equipment that comes along.

As an example, the MoM includes the following components (Figure 1):

1. A robust plastic box, we use a Peli Case 


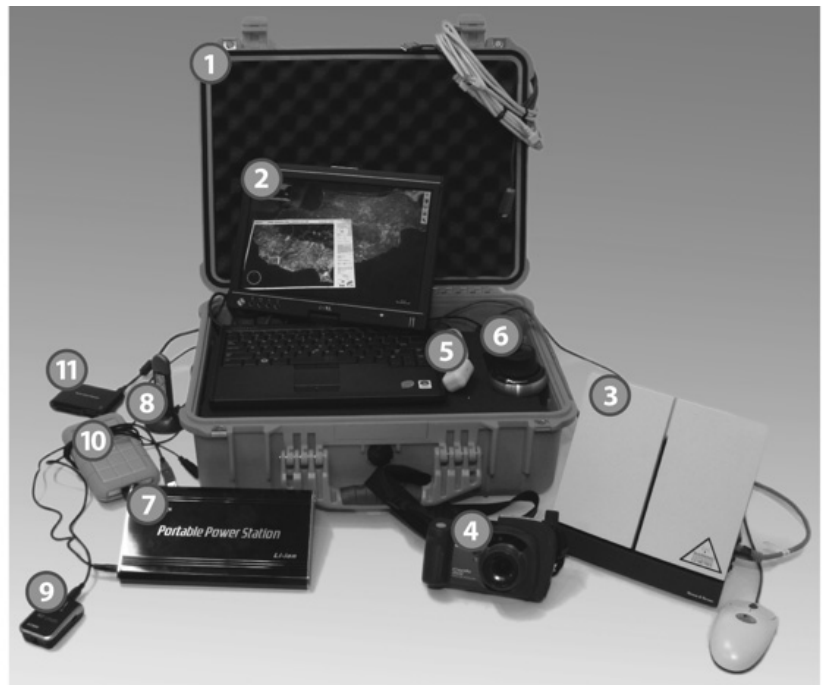

Figure 1: Mobile mapcase (MoM).

2. A semi-rugged convertible notebook with touchscreen

3. A BGAN terminal for satellite communication

4. A digital camera (with build-in GPS and WLAN) for georeferenced photography

5. A 3D compass module to align the BGAN terminal and to orient the map view

6. A 3D mouse to navigate in a three dimensional space [optionally]

7. An external power pack to extend the runtime

8. An external WLAN antenna

9. A GPS module for positioning

10. Some external memory (stick or harddrive) and a

11. card reader for data import, export and storage

\subsection{User interface}

The whole system has to be intuitively usable by the user, letting him do his original work. It has to support the user, not slowing him down (rather speed him up) or distract him too much, which is often the case with other systems tested by the interviewed relief workers. The users are not necessarily experts in using their equipment, they might use it just once a year, so it is even more important to follow the minimum-configuration approach and to offer an intuitively usable user interface.

To provide an easy access to external parties like the Monitoring and Information Center (MIC) in Brussels we provide a web start version of the software, 
which can be started by simply clicking a link on a web page. After having it downloaded, the software can also be started in offline mode.

\subsection{Mapping}

As described beforehand, maps and especially satellite based rapid mapping is valuable information for relief workers. In many cases it is the only information available for a basic situation assessment. Today, it's a challenge to bring the maps into the field and to keep them updated: They are transmitted using common ways (email, download from a website or ftp server) and then printed out using portable printers, a manual process which needs time and effort. It would additionally be a great benefit to bring back information from the field to the map producers, to help them clarifying the situation and improve updated map versions.

In our "electronic mapcase", maps are made visual in an electronic form, embedded in a 3D globe, fully navigable and zoomable by the user. Also some basic operations like changing the transparency or the order of the maps are possible. Using the networking technologies described later, the maps can be automatically distributed and it is easily possible to update the maps if newer versions are available.

Based on an image file, the procedure to import maps includes an automatic tiling of the image, to increase the performance of the 3D application. The needed meta data to georeference the map has to be available in standard formats such as World Files or KML files. The map injection (i.e., the import into the system) can be done directly by the map producers, for example the ZKI (http://www.zki.caf.dlr.de), or manually by the user, who could have downloaded the map from a web page. After it has been injected by one user, it will be automatically distributed to other mapcases, depending on the network availability. If a newer version is available, it can automatically be replaced by the older version. Another possibility for map import is to use Web Mapping Servers like WMS (http://www.opengeospatial.org/standards/wms), although it is necessary to have an internet connection in this case. However, after the data has been loaded into the system, it can also be distributed via other networks.

It is also very easy to return information from the field back to the map producers. This information can for example be georeferenced photos, which bring a better impression about the real situation in the field to the producers, who can then refine their maps in future versions.

\subsection{Networking/communications}

Especially in networking, robustness, fail-safe and minimal-configuration are important objectives. The network management has to be organized by the system, again insulating the user from configuration effort, though this is not always possible. Sometimes a user interaction is necessary, if for example an antenna has to be pointed or a password for WLAN access has to be entered. 
In disaster situations, one can not rely on existing infrastructures. To be independent from those, we use a combination of ad hoc and infrastructured networking, to be able to either use still existing, conventional networks but also to form a new, self-organizing network if no sufficient communication means are available. Using several network devices and different communication technologies, redundancy is created and more possibilities are available, which increases the probability of available communication links.

An essential part is satellite communication: It is highly reliable and deployable everywhere (in the range of the satellites), it works also if local communication infrastructure is not existent, proprietary, or if it is directly affected by the disaster. But also other technologies have to be considered, as satellite links are expensive to use and limited in their bandwidth.

We use short range communication to directly synchronize the mapcases. For the use in the field, there is no need for a communication infrastructure. If data (like maps) has to be imported from outside the locally available mapcases, it is sufficient to transmit it once (e.g. via the expensive satellite link) and then distributed using the short range networks.

Additionally, all data is cached locally, so it's possible to use broadband communications to preload maps and other data and to have it available if needed at a later time.

\section{Implementation}

The system is implemented using the Java programming language, in order to make the software easily deployable for several operating systems. We use the 3Dapplication framework NASA World Wind (http://worldwind.arc.nasa.gov/java/ index.html), which already includes a lot of functionality and allows us to fully adapt it to our needs. We also use open source libraries wherever possible, like apache commons (http://commons.apache.org/) or the apache mina FTP server (http://mina.apache.org/ftpserver/), as well as standard data formats. All together, we are aiming at having the possibility to distribute the system as easy as possible for a low-cost end user price.

\subsection{BGAN pointing wizard}

For most non-expert users, the pointing of the antenna is one of the biggest problems when using a satellite network connection. This was the reason for us to develop a pointing wizard, which shows the user in which direction to move the antenna and when the field strength of the satellite signal is sufficient to connect. For this we use a 3D compass, which is able to determine it's attitude in the threedimensional space. Together with the position of the mapcase, the information can be used to calculate the bearing for the antenna and to make this information available using an intuitive graphical interface (see Figure 3). 


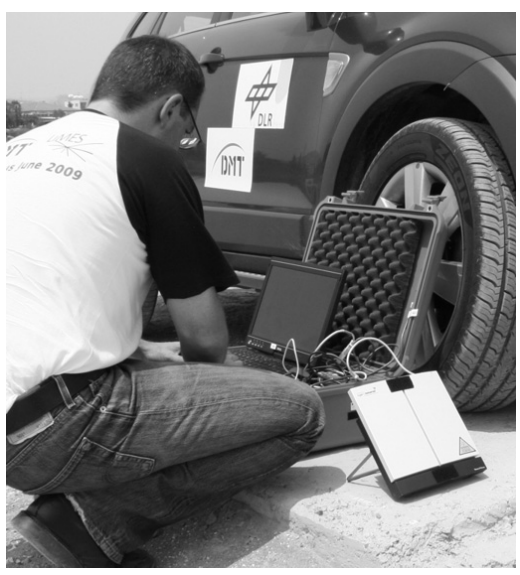

Figure 2: Pointing the antenna.

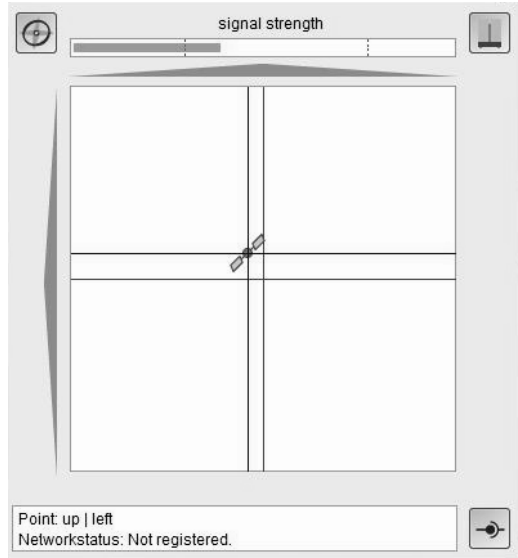

Figure 3: Pointing wizard.

\subsection{POIs and assessment reports}

A main source for data import are points of interest (POIs), attached with reports or pictures. When the user takes a photo and uploads it via the camera's WLAN link to the mapcase, a new POI can automatically be created and additional information can be added. To help the user searching for the right information when assessing the situation, we use report forms, based on drafts used by the United Nations Joint Logistics Center (UNJLC) and other relief organizations. These forms can be easily changed as they are rendered using a simple XML (eXtended Markup Language) structure, so the user (or someone in the headquarters) can add his own reports or change existing ones to adapt them to the current situation.

Every POI has a type, the basic ones we have included so far are airport, bridge, harbor, hospital, power plant, radio station, railway, road, warehouse and a default POI. The POIs are also shown in the map view (see Figure 4), can be easily accessed and are distributed automatically.

\subsection{Communications}

The internal communication and synchronization can be used independently from the physical network connection, with different functionality depending on the characteristics of the underlying network.

For long range communication, we use the Inmarsat satellite network with a BGAN terminal. For short range, we use WLAN, utilizing TCP/IP as well as a self developed protocol, the Disaster Management Tool Protocol (DMTP). DMTP is used to detect other entities in the range of the antenna, to connect them and to exchange data. Via DMTP, network connection data like the IP addresses can be exchanged to be able to connect via other (faster and more robust) networks. Also payload data can be transmitted (i.e. broadcasted in the antenna range) via DMTP. 


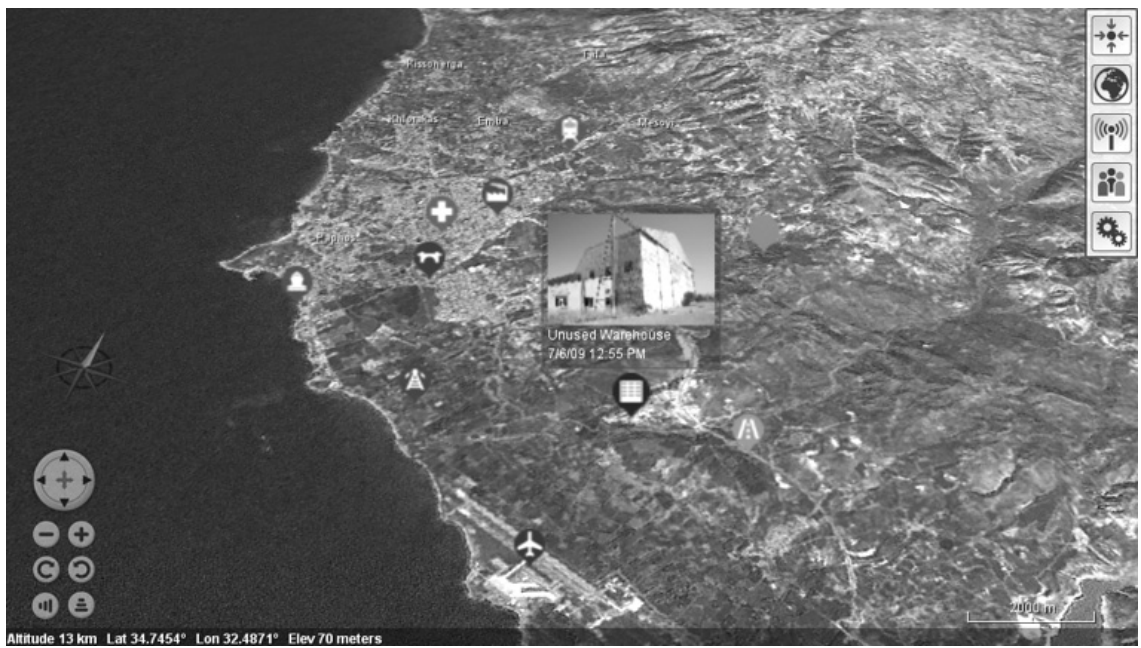

Figure 4: Map of south west Cyprus including some real and simulated points of interest for illustration.

We have two WLAN adapters running, one to connect to an ad hoc network for the digital camera and other mapcases in range using TCP/IP and DMTP at the same time and a second one to connect to infrastructured networks, if available. Of course, the use of wired LAN is also possible.

\section{Experimental results}

\subsection{Constraints and scenario}

The main evaluation criteria is the usability and added value of the demonstrated system for the relief workers.

In order to test and demonstrate the possibilities of the DMT, we had the chance to take part at the European Commission's Assessment Mission Course (AMC), that is held regularly in Cyprus. The main objective of the AMC is to train experienced disaster managers of the EU's Member States and other international organizations in planning and conducting assessment missions within and outside the EU. An additional objective is the development of a European assessment methodology, which can be shared with other national and international organizations.

The course/exercise is based on an earthquake scenario and focusses exclusively on the assessment phase (not relief). Participants are tasked to perform damage and need assessments in urban and rural areas as well as at industrial and logistically important sites. Key sectors to be assessed are search and rescue (SAR), shelter and important infrastructures like electricity supply or transportation and logistics. 
Through the support of the Cyprus Civil Defence, which functions as the Local Emergency Management Agency (LEMA) throughout the exercise, participants will have the opportunity to meet with and interview the actual holders of key functions in Cypriot authorities on federal, district and municipal level emergency management and representatives of industrial and logistical installations (airports, harbours, etc.).

Different kinds of maps that were modified according to the disaster scenario were prepared by DLR and SERTIT (http://sertit.u-strasbg.fr). The Mapcase concept and hardware was presented to the AMC organizers and trainees in the preparation day prior to the exercise. During the course days some of the teams had the possibility to take a Mapcase with them during their assessment missions. One Mapcase remained at the local headquarters to allow transmitting and receiving information from and to the teams. The system was used to generate situation assessments and to provide operation support information.

\subsection{Results}

In general the user feedback given during the course and at the debriefings was quite positive. Especially the added value of satellite map products was highlighted. The demonstrated mapcases were evaluated as an innovative and helpful way for improving the speed of exchanging information between the different actors during disaster relief operations. The variety of useful services and features that are provided by the Mapcases and the availability of all these services in one box was one of the main advantages seen by the course participants.

Some additional feedback is summarized below:

- The "fully-integrated" approach that provides one user interface for map viewing, entering assessment reports, communication setup and automatic geo-referencing has been highly praised and is seen as the key differentiating feature to other systems

- The integration of voice and video communication/conferencing between Mapcases within the user-interface is not as favored as we thought, since other, commonly used communication systems are preferred.

- The BGAN pointing wizard allowed successful pointing by non-experts within very short time

- The current weight of the Mapcases is acceptable, but the system should be merged with already existing equipment (like EU's Assessment Mission Kit).

- The capability for not directly involved persons (e.g. in Brussels) to get an overview over the situation by using the web start version was seen as a very good feature.

\section{Conclusions}

An integrated Disaster Management Tool (DMT), adapted to the special needs of international relief organizations has been designed, built and tested. The 
initial motivation was the lack of a tool that combines individual components to an integrated, rapidly deployable and easy to use system which can bring a satisfactory add-on value to the disaster management procedures. Communication and navigation support, disaster assessment support and information management are the core key functionalities covered by the DMT.

Potential users have been involved in the design process from the very first stage and the outcome is a system which is approved for its usability and usefulness. The system was tested in several humanitarian relief exercises and the feedback which has been collected helped us a lot to bring the system into its current stable and mature stage. The development chain of the DMT is a continuous process with more advanced features and technology alternatives to be added, more user feedback to be considered and cost reduction strategies to be applied. This will help in bringing our system to the state of being useful for any relief organization in any disaster scenario.

The system is suggested to be used in upcoming training exercises and presented to a broader audience at EU as well as UN events.

\section{Outlook}

Our future development plans are mainly based on user feedbacks and technology requirements.

Our graphical wizard for adding maps will be extended to allow the injection of non geo-referenced maps and to reference them on the fly. For example, a printed version of any map could then be photographed and added to the system.

Some improvements are ongoing in the area of information management and security. Different data access rights should be assigned to the several layers of the humanitarian relief chain. Managing more than one disaster situation should be possible and data sets should not get mixed. Improved unit management functionalities will be added to have a better overview of the units and their states, enhancing the management of operations.

Normally, the available infrastructure and resources varies according to the type, extend and location of the disaster and the organizations involved. Accordingly, work on providing different options of communication and navigation technologies, information management and synchronization methods is in progress. For example, TETRA and DVB-RCS are currently being tested to provide communication alternatives.

We expect our DMT to be used also in environments where the number of visible navigation satellites is insufficient for positioning. Accordingly, investigations on integrating our Indoor/Outdoor Positioning System [5] into the DMT is in progress.

Some major ongoing block of work is the development of the Wearable Units (PAMs). A PAM consists of Mobile PC with internal WLAN adapter, a (helmetmounted) digital camera, an inertial measurement unit for pose tracking and a GPS Module. The PAM will provide just a subset of the DMT's functionality to achieve improved mobility. 


\section{References}

[1] Hüls, E. \& Oestern, H.J., Die ICE-Katastrophe von Eschede - Erfahrungen, Lehren, Konsequenzen. Notfall \& Rettungsmedizin, 2(6), 1999.

[2] von Kirchbach, H.P., Bericht der Unabhängigen Kommission der Sächsischen Staatsregierung Flutkatastrophe 2002.

[3] Hurricane Katrina - A nation still unprepared, 2006.

[4] Cannizzaro, G., Bocci, L. \& Proietti, S., LBS Applications \& Services to support Security management in humanitarian relief, 2006.

[5] Wendlandt, K., Khider, M., Angermann, M. \& Robertson, P., Continuous location and direction estimation with multiple sensors using particle filtering. Proc. 2006 International Conference in Multisensor Fusion and Integration for Intelligent Systems (MFI 2006), Heidelberg, 2006. 